\title{
Explotación sexual de niños, niñas y adolescentes. Comprender para transformar. Estudio de las prácticas de trabajo con supervivientes realizadas puestas en marcha por una asociación de protección a la infancia en Madagascar
}

\author{
Olga Bautista Cosa ${ }^{1}$
}

\section{Recibido: 30/11/2016 / Revisado: 21/02/2017 / Aceptado: 28/11/2017}

Resumen. Este artículo muestra los resultados de una investigación llevada a cabo en un recurso especializado de atención a jóvenes en situación de explotación sexual, en Madagascar. La explotación sexual ha tomado en las últimas décadas una dimensión y unas características que son propias de esta época globalizada. La relevancia de la problemática requiere un marco teórico sólido y bien armado que permita poner en marcha acciones pertinentes que ayuden a superar y transformar la realidad que fomenta y mantiene esta problemática.

La investigación que se ha llevado a cabo pretende contribuir a la creación de conocimientos significativos en torno a esta temática, incorporando los conocimientos y marcos de trabajo más relevantes en la materia, las prácticas de los profesionales y las experiencias de las y los jóvenes.

Palabras clave: Explotación sexual; prostitución juvenil; intervención social; transformación social; perspectiva de género, Madagascar.

[en] Sexual exploitation of boys, girls and adolescents. Understanding to transform. Study of work practices with survivors implemented by a child protection association in Madagascar

\begin{abstract}
This article shows the results of research carried out with a specialised resource offering care to young persons in situations of sexual exploitation in Madagascar. In recent decades, sexual exploitation has assumed a dimension and characteristics that are reflective of this globalised era. The significance of the problem requires a solid and well-designed theoretical framework that permits the implementation of pertinent actions to help overcome and transform the reality that foments and maintains this problem.

The research conducted is aimed at contributing to the creation of meaningful knowledge in this area, incorporating the most important knowledge and working frameworks in the field, as well as professional practices and the experiences of the young persons involved.

Key words: Sexual exploitation; youth prostitution; social intervention; social transformation; gender perspective; Madagascar.
\end{abstract}

Sumario: Introducción. 1. Metodología. 2. Resultados. 2.1. Con respecto a los y las profesionales. 2.2. Con respecto a las jóvenes. 3. Conclusiones. 3.1. Con respecto al marco de trabajo. 3.2. Con respecto al programa de intervención. 4. Recomendaciones. 5. Referencias bibliográficas.

Cómo citar: Bautista Cosa, O. (2018) Explotación sexual de niños, niñas y adolescentes. Comprender para transformar. Estudio de las prácticas de trabajo con supervivientes realizadas puestas en marcha por una asociación de protección a la infancia en Madagascar, en Cuad. trab. soc. 31(2), 517-527.

\footnotetext{
Médicos del Mundo, Francia

olga.bauco@gmail.com
} 


\section{Introducción}

La industria del sexo se presenta, en la era de la globalización, como una problemática que se extiende a nivel mundial de forma interconectada y cambiante. Hablamos de industria del sexo para referirnos a todos los productos de consumo (incluyendo a las propias mujeres, jóvenes y niñas, a una parte de ellas, y a las imágenes derivadas de sus cuerpos o de actos sexuales donde se han visto implicadas); a todos los lugares donde se comercializan estos productos (desde las salas de masajes con final feliz, pasando por agencias, bares de striptease, páginas web de visionados de contenido pornográfico, etc.); y a la prostitución de supervivencia, donde las mujeres y jóvenes obtienen ayuda, en casos de necesidad u urgencia a cambio de servicios sexuales (alojamiento en caso de fuga del domicilio familiar, alimentación, reparaciones en la casa, pago de la matrícula escolar, comida, etc.). Esta definición corresponde a una descripción amplia de la industria e incluye tanto situaciones donde ha habido elección como situaciones donde las mujeres y jóvenes han sido forzadas de algún modo o se encuentran dentro de una red de trata. En el caso de los niños y niñas no podemos hablar de elección en ningún caso (Szczepanick, Ismé y Boulebsol, 2014).

En este sentido la explotación sexual infantil y adolescente (ESIA) es una manifestación más de la industria del sexo mundializada, uno de los signos de identificación del modelo económico neoliberal globalizado de las últimas décadas (ATTAC, 2009).

Se habla de explotación sexual cuando existe trata de niños, niñas y adolescentes con fines de explotación sexual; cuando hay una utilización de niñas, niños y adolescentes en relaciones sexuales remuneradas ( con dinero, en especie: ropa, comida, regalos- o por medio de favores: por ejemplo, que les pongan buenas notas en el colegio) incluido el llamado turismo sexual o explotación sexual infantil en los viajes; y cuando nos encontramos ante imágenes -sean reales o no- de abuso sexual de niños, niñas y adolescentes en internet (Infancia, 2010).

Como problemática, que afecta de forma mayoritaria a las mujeres y a las niñas en el 89 por ciento de los casos, según algunos estudios (Orjuela López y Rodríguez Bartolomé, 2012), el feminismo se ha interesado por el fenómeno de la industria del sexo y la prostitución.
Así, se han perfilado en las últimas décadas dos posturas aparentemente irreconciliables: a) la abolicionista, que considera la prostitución y la industria del sexo en general como una forma de violencia de género, basada en las relaciones históricamente desiguales entre hombres y mujeres, que refuerza la hegemonía sexual masculina, la división tradicional del trabajo, donde mujeres y jóvenes no tienen capacidad de elección; y que propone como solución la penalización del cliente. b) la reguladora, que considera la prostitución y la industria del sexo como un tipo de trabajo más, que expresa la libertad y la posibilidad de elegir de muchas mujeres respecto a su sexualidad y a su cuerpo; que supone una forma de búsqueda de autonomía, que no está basada en una desigualdad de género y cuyo único problema es el estigma, que se solucionaría con una regulación de la actividad que permitiría acceder a ciertos derechos básicos a las mujeres implicadas en esta actividad. Otras dos posturas, fuera del feminismo, también se han sumado al debate: a) la que considera la prostitución como una manifestación de que las mujeres que la ejercen están enfermas; b) la que, basada en teorías freudianas, ve en la prostitución una manifestación de la sexualidad desviada de las mujeres que se prostituyen. En ambos casos se considera que las mujeres pueden ser tratadas de forma individual, curándolas y rehabilitándolas (Levin y Peled, 2011).

Según la manera de interpretar la problemática, las medidas pensadas y puestas en marcha para erradicarla son radicalmente opuestas y colocan a las mujeres y a las jóvenes implicadas en lugares muy diferentes.

La presente investigación apuesta por una postura hibrida entre la postura abolicionista y la postura reguladora, tal y como propone Beatriz Gimeno en su libro, La Prostitución. Apuntes para un debate abierto (2012). Así se considera la industria del sexo en todas sus manifestaciones como un tipo de violencia de género basada en las relaciones históricamente desiguales entre hombres y mujeres, así como en las desigualdades y opresiones basadas en la clase, la raza y la orientación sexual, que se manifiesta histórica y socialmente de una manera determinada en cada momento y sociedad dados. Las soluciones propuestas pasan por trabajar en la construcción de sociedades más igualitarias donde las personas tengan acceso a la información y formación adecuada sobre salud afectivo-sexual y donde se les reconozca 
como agentes con capacidad de decisión y que se les ponga en el centro de las políticas de intervención para atender a sus necesidades, ya sean estas continuar o salir de la industria del sexo; así como proponer sistemas de protección y autoprotección ante otras posibles violencias que puedan estar sufriendo, siendo el objetivo final la puesta en marcha de sistemas sociales y comunitarios que construyan unas sociedades libres de explotación. Esta idea de poner en el centro está avalada por organismos internacionales como Naciones Unidas con su Declaración de Beijing de 1995 o el Informe del Comité Fraser sobre la industria del sexo, realizado en Canadá en los años 1980 ( citado en Gimeno, 2012, pp.286 y 267) .

Con este marco de interpretación, la explotación sexual de niños, niñas y adolescentes se define como un tipo de maltrato infantil (ECPAT International, 1996); un tipo de violencia de género (ONU, 1995); como la manifestación más extrema de la violencia sexual contra niños, niñas y jóvenes (Orjuela López y Rodriguez Bartolomé, 2012) y como una violación de sus derechos.

Desde un punto de vista macro, la explotación sexual infantil y adolescente forma parte de las diferentes dinámicas de construcción de intercambio de capitales y de la construcción de subjetividades de la lógica neoliberal, nutriéndose y alimentándose de las siguientes áreas de nuestra realidad social: neoliberalismo y sociedades mercantilistas: idea de que todo es susceptible de compra, el consumo es rápido y efímero, el cuerpo se contempla como un soporte para el mercado y no como un soporte para la vida; relaciones de poder: patriarcado, sociedades adultrocéntricas, desigualdades entre Norte y Sur; estigma e invisibilidad; sexualidad normativa y discriminatoria; violencia y dominación simbólica (Bustamante, 2010)

La invisibilidad a la que se enfrenta la explotación sexual infantil y adolescente tiene como consecuencia una negación y un no-reconocimiento de su existencia. La falta de acuerdos en torno a la terminología para describir y nombrar la temática, al igual que un corpus teórico todavía en construcción contribuyen igualmente a esta problemática (Idem).

En este sentido, el African Partenership to Violence Against Children, grupo de trabajo e incidencia política que lucha para erradicar todas las manifestaciones de violencia contra niños, niñas y jóvenes, cita a Unicef para comentar que, de todas las violencias presentes en
África, la explotación sexual infantil y adolescente es la que mayor incidencia tiene en este continente; siendo una de las zonas del planeta más afectada por esta problemática. Señalan también que existen pocos conocimientos sobre las particularidades de la explotación en el contexto africano y desde el mismo. (Maina Wanjiru, 2016)

La investigación propuesta vendría a aportar nuevas perspectivas sobre este fenómeno, haciendo un análisis crítico, basado en el modelo hibrido enunciado anteriormente, de las prácticas de intervención que actualmente se llevan a cabo en el contexto de Madagascar, país elegido para realizar la investigación.

Madagascar cuenta con una población estimada de 23.571.713 personas. De estas, 10.000.000 son niños, niñas y adolescentes, de los cuales 8.200.000 viven bajo el umbral de la pobreza.

En este contexto de vulnerabilidad y precariedad, la situación de las niñas y las mujeres es especialmente alarmante con: un aumento de la feminización de la pobreza, un acceso muy precario al sistema de salud y a una información adecuada sobre salud sexual y reproductiva; la legitimización de la violencia de género de manera general (el 45 por ciento de las mujeres y de las jóvenes considera normal la utilización de la violencia de los hombres sobre las mujeres); y de la violencia sexual en particular ya que la ley del matrimonio civil sigue otorgando al marido el derecho a mantener relaciones sexuales con su cónyuge aunque esta no lo desee (Ravaozanany, Rakotoarindrasata, De Dieu, Rabenoro y Rasendrasoa, 2012).

El país es víctima de crisis políticas y económicas recurrentes, desde los años 1980. La más reciente, en 2009, volvió más precaria aún la economía, haciendo desaparecer casi los pocos servicios que presta el Estado y empeorando significativamente la calidad de vida y las libertades de la población en general. Un repunte significativo de la explotación sexual infantil y adolescente han sido observados por los actores de terreno desde ese año, y Madagascar empieza a conocerse como la nueva Tailandia (Buisset y Doppler, 2013).

El estado malgache ha ratificado numerosos convenios internacionales, que ha concretizado con la creación de leyes específicas a nivel nacional para luchar contra este problemática. Estas medidas no han sido lo suficientemente efectivas. La falta de un diagnóstico claro sobre las causas profundas que persisten tras el 
fenómeno, la debilidad y corrupción de las instituciones, el fraccionamiento de la sociedad sobre todo en los núcleos urbanos (Ricaldi Coquellin, 2010) - y la tolerancia social debilitan cualquier acción que se haya llevado a cabo en este sentido los últimos años. (Buisset y Doppler, 2013). Así, la manifestación más común de la explotación sexual de niños, niñas y adolescentes que se observa es la prostitución de supervivencia, ya sea con la población local o con turistas internacionales (Idem).

Los factores que aparecen como determinantes para las chicas y chicos, a la hora de acceder a la prostitución en este contexto, son principalmente dos:

- La cobertura de necesidades básicas: comida, educación, ropa, etc. (D.CH.S., 2016).

- La búsqueda de un ascenso social mediante el matrimonio con un extranjero (Cole, 2010)

En ambos casos se trata de estrategias que buscan la supervivencia y un cierto grado de autonomía. Estos supuestos contradicen la idea de que el o la adolescente no tiene ningún tipo de poder de decisión en estas circunstancias. Si bien se trata de una elección muy restringida, no deja de ser una toma de iniciativa en búsqueda de una mejora de vida en una sociedad con unos patrones muy marcados y con pocas alternativas a los modelos de acceder a ser una mujer o un hombre adultos; es decir, con capacidad de elección sobre su propia vida (Lagarde y de los Ríos, 1990).

La literatura existente sobre le materia se centra en su gran mayoría en analizar las causas de la problemática. Así lo confirma la investigación que ha llevado a cabo la organización canadiense, Concertation des luttes contre l'exploitation sexuelle (CLES), sobre las necesidades de las jóvenes y adultas que desean salir de la industria del sexo. Según las conclusiones del estudio, muy pocos trabajos de investigación han abordado la problemática desde este punto de vista. Otra de las conclusiones a las que llega el estudio es que poner en marcha políticas que no tienen en cuenta lo que las mujeres necesitan conduce a realizar iniciativas no adecuadas, tendentes a re-victimizar a las jóvenes y que pueden dificultar la salida de las mujeres de ella (Szczepanick, Ismé y Boulebsol, 2014).

Documentan estas autoras tres modelos propuestos para la intervención en explotación sexual. El modelo de Mansson y Hedin (1999), que se basa en el capital social de las mujeres para movilizar a su entorno (familiares, amigos). El éxito de la salida depende de las capacidades de las mujeres y de su entorno, no se incluye ningún análisis de factores sociales que pueden influir en este proceso. El modelo de Baker (2000), basado en los cambios en la identidad y en la vida de las mujeres que toma en cuenta las circunstancias individuales que influyen en el proceso de cada mujer. Y el modelo de Cimino (2012), basado en la consideración de las circunstancias individuales de cada mujer y su influencia en la intervención, así como en el análisis de las influencias exógenas -como la pérdida parcial de la capacidad de agencia- a tener en cuenta en el diseño y puesta en marcha de la intervención. Ninguno de los tres puede generalizarse, ya que analizan tan solo el fenómeno de la prostitución de calle y no incluyen ningún análisis de las relaciones de poder y dominación que permiten la aparición y el mantenimiento de la problemática.

Destacamos la propuesta realizada por Skrobanek, Boonpakdi y Janthakeero (1997), en el contexto de Tailandia, como la única que propone dar un rol principal a la investigación y a la intervención, desde una óptica feminista y participativa, y a la implicación en la misma de las mujeres y jóvenes involucradas en la industria del sexo para poner en marcha acciones basadas en evidencias y lideradas por las propias mujeres. En este sentido el modelo se puede acercar más al modelo hibrido, del que habla Gimeno.

La investigación propuesta pretende alimentar esta línea de trabajo y creación de conocimiento. Si bien como hemos dicho, esta problemática toma formas distintas, según la parte del mundo donde se produzca, el análisis que se lleva a cabo podrá ayudar a establecer pistas de reflexión y recomendaciones de trabajo con las supervivientes ${ }^{2}$ adaptables a otros contextos. En este sentido, se ha elegido realizar un análisis de las acciones puestas en marcha por una estructura de protección a la infancia y defensa de derechos de los niños y

La elección de la palabra "superviviente" en vez de "victima" corresponde también con el marco de análisis de la problemática propuesto. Asi, las mujeres, adolescentes y niñas son situadas como agentes de sus propias vidas. 
niñas en materia de explotación sexual infantil y juvenil, que lleva trabajando en Madagascar desde hace más de 10 años. En los siguientes apartados abordaremos la metodología utilizada en la investigación y las principales conclusiones y recomendaciones a la que se ha llegado.

\section{Metodología}

La metodología de investigación elegida es de carácter cualitativo y bebe de los principios de la metodología comunicativa crítica (Gómez, Latorre, A., Sánchez, M. y Flecha, R., 2006) y de la metodología constructivista.

Se ha seleccionado especialmente la metodología comunicativa critica por su capacidad para permitir un análisis de las problemáticas sociales que favorece su transformación, mediante la identificación de aquellos componentes que generan exclusión y aquellos que contribuyen a la transformación de las desigualdades. Se trata, además, de una metodología que ha demostrado tener impacto científico y social en diferentes investigaciones destacadas y ha sido validada, a nivel internacional, a través de su publicación en un Special Issue de la revista Qualitative Inquiry, primer cuartil del área de conocimiento Social Science Interdisciplinary del ranking Journal Citation Report.

Además, se han incluido principios de las prácticas de investigación feminista, y con víctimas de violencia de género. De las epistemologías feministas, rescatamos las propuestas realizadas por los feminismos negros (Jabardo, 2012) y decoloniales (Bidaseca y Vazquez Laba, 2011), rescatando las ideas de conocimiento situado de la persona que realiza la investigación, así como el análisis multifactorial de las diferentes opresiones que nos atraviesan como personas - clase, raza, genero- para abordar la problemática.

Los marcos de trabajo elegidos apuestan por la puesta en relación de la literatura existente sobre el tema, las entrevistas con profesionales y el estudio de historias de vida. Entran además en contacto con la realidad que analizan, creando nuevos conocimientos que permiten cuestionar y transformar esa misma realidad; cumpliendo así con el objetivo de poner la investigación al servicio de las sociedades donde se integra.
La selección de la estructura de protección correspondió a dos criterios: la especialización en la temática y los años de trabajo en terreno. Todo el personal técnico de la estructura -7 educadores/educadoras sociales, una médico y un antropólogo- han participado en la investigación. La selección de las jóvenes se ha realizado de acuerdo con el equipo de la asociación, siguiendo criterios de estabilidad psicológica y recuperación de cada una de ellas, para evitar la victimización secundaria en la realización de las entrevistas. Las zonas donde se circunscribe la investigación corresponden a las zonas urbanas del país donde está presente la asociación con la que se ha trabajado: en Antananarivo, la capital y Nosy $\mathrm{Be}$, uno de los principales destinos del turismo internacional.

La investigación no pretende realizar un muestreo cuantitativo que obtenga una muestra extrapolable, sino analizar un marco, una metodología y unas técnicas de intervención, así como la manera en que las mismas han impactado en la vida de las jóvenes y los jóvenes participantes en el programa, para seguir visibilizando la realidad ligada a la explotación y poder ofrecer un marco de reflexión para futuras investigaciones e intervenciones.

La investigación se ha desarrollado en 4 fases. La primera, consistente en la revisión de las revistas indexadas en el Journal Citation Report de 2015, en las categorías de Womens studies y Social Work; y la consulta de publicaciones e investigaciones sobre la temática a nivel internacional. La segunda, de elaboración del cuestionario para los profesionales, la guía de entrevista para las jóvenes y el documento de consentimiento informado, según los modelos propuestos por Adams, Ahrens, Campbell, Self y Wasco, (2009) y Levin y Peled, (2011). La primera propuesta busca poner de manifiesto las voces de las jóvenes supervivientes. La segunda apuesta por averiguar las percepciones de los y las profesionales respecto a la problemática. La decisión de utilizar técnicas diferentes con las jóvenes y los/las profesionales corresponde a una búsqueda de informaciones de naturaleza diferente en cada caso. La tercera fase, correspondiente al trabajo de campo, con la elaboración de 9 test auto-administrados con profesionales, 1 entrevista a un profesional $^{3}$ y 3 entrevistas semiestructuradas a jóvenes supervivientes. Y la cuarta, sobre la 
puesta en diálogo de los resultados obtenidos en el trabajo de campo con los resultados obtenidos en las lecturas de literatura científica y redaccion de las recomendaciones para futuras intervenciones.

Se ha respetado las indicaciones de ética deontológica del trabajo de investigación con poblaciones vulnerables, siguiendo los principios recogidos en el Código Ético de la Asociación Internacional de Sociología (ISA, 2001), así como en el proyecto ERIC (ERIC, 2013).

Para la realización de las entrevistas con las jóvenes se han seguido las recomendaciones de la Organización Mundial de la Salud (OMS, 2001) en lo que se refiere a la elaboración de investigaciones sobre violencia de género:

- Seguridad y confidencialidad

- Traducción de las herramientas y puesta a disposición de una interprete bilingüe francés-malgache

\section{Resultados}

\subsection{Con respecto a los y las profesionales}

El marco de análisis corresponde a una estructura: el Centro de día de carácter abierto para chicas y chicos en situación de explotación. La estructura se compone de 9 profesionales que trabajan de forma directa con las chicas o con la elaboracion de materiales pédagogicos y/o de investigacion. Se distribuyen de la siguiente manera: 5 educadores/as en Antananarivo y 2 en Nosy Be; una médico y un antropólogo, en Antananarivo. El objetivo final del recurso es reincorporar a estas jóvenes en la sociedad a través de su inserción laboral.

Los test auto-administrados han permitido obtener información del equipo en relación a los siguientes puntos:

- Percepción de la explotación: los resultados de la escala de medición de Levin y Peled (2011), muestran que el equipo percibe la explotación y la prostitución como una forma de violencia que debe eliminarse. Muestran además una persistencia de ciertas creencias como: la consideración de que se trata de la manifestación de un deseo se- xual desviado; que es una violación donde a la víctima se le paga; que es un foco de trasmisión de VIH/Sida: o que las jóvenes no son capaces de salir de la situación de explotación donde se encuentran.

- Formación previa: este indicador ha permitido evaluar el nivel de formación previa al inicio de la intervención sobre la temática. Esta es una de las recomendaciones para el trabajo con víctimas de violencia. (OMS, 2001) Tan solo 5 de las 9 personas pertenecientes al equipo tenían algún tipo de formación que les habilitara a ejercer su puesto de trabajo. La formación previa sobre explotación no incluía ningún contenido sobre derechos humanos, marco legislativo, género o violencia de género, trauma o atención a víctimas de violencias.

- Marco de interpretación de la explotación sexual: este ítem ha permitido evaluar qué marco ideológico y de interpretación se aplica para el análisis de la problemática en el recurso. Con respecto a los/las explotadoras, no establecen ningún tipo de distinción entre géneros y se asocia el consumo de prostitución con la expresión de un deseo irrefrenable y/o con el consumo de drogas. A las y los jóvenes se les considera víctimas de una situación que no han podido elegir. Los problemas familiares o personales previos aparecen como las únicas causas de entrada en la industria del sexo. Estos problemas pasan por: el divorcio de los padres, la monoparentalidad, la prostitución de la madre, el consumo de drogas o la falta de comunicación, la homosexualidad, vista para ellos como una enfermedad, y la hipersexualidad, considerada para ellas como ninfomanía. La explotación se analiza como un hecho ahistórico y fuera de contexto, y sin conexión con otras violencias o con otras manifestaciones de la industria del sexo. Solo existe explotación cuando las personas son menores de 18 años; cuando se considera que no pueden dar ningún tipo de consentimiento para participar en la actividad. No se tiene en cuenta criterios de psicología evolutiva o el contexto del país 
donde el trabajo infantil está normalizado como un marco válido para comprender la postura de los y las jóvenes.

- Intervención: este indicador nos ha permitido analizar las diferentes prácticas desarrolladas por el equipo. Con respecto a la selección de los y las jóvenes se distinguen dos etapas: de 2009 a 2012, cuando era requisito indispensable para la entrada en el recurso el examen médico sobre el estado de salud de la joven que incluía una prueba de embarazo y una prueba de virginidad para determinar si efectivamente la chica era activa sexualmente, lo que siguiendo el imaginario colectivo tradicional de Antanarivo, equivale a que ejercía la prostitución. De 2012 a la actualidad, se realiza una pre-identificación en el trabajo de calle. Los chicos adolescentes se toman en cuenta. Los criterios de entrada están en relación con la frecuencia de la práctica por los y las jóvenes. Un mayor número de veces por semana equivale a considerarlos/as automáticamente como víctimas. Con respecto al circuito de intervención, una vez dentro del programa, es un modelo preestablecido que no se negocia con las/los jóvenes. Con una duración de un año y medio, incluye un seguimiento psicológico, una formación en realfabetización y en francés, la participación en una formación profesional (cocina o peluquería) y la búsqueda de empleo o creación de autoempleo (pequeños negocios en la economía informal como venta ambulante, pequeños quioscos de venta de comida o ropa), así como el derecho a asistencia sanitaria, la participación en talleres de educación artística y la sensibilización en materia afectivo-sexual con otros recursos especializados. Con respecto a este servicio concertado, los profesionales refieren haber tenido dificultades:

Algunas chicas...vinieron a quejarse después de ponerse el implante...Nosotros no las obligamos a ponérselo solo se lo aconsejábamos vivamente...en fin que vinieron a quejarse porque nadie les había dicho que la duración era más larga que el tiempo de estancia en el recurso... y querían quedarse embarazadas y no tenían dinero para quitarse el implante....convencimos a la asociación de que pagase la retirada aunque ya no estuvieran a nuestro cargo... decidimos ir con ellas a la consulta del médico porque le tenían miedo, les echó la bronca cuando fuimos por no haberse informado bien antes, pero eran ellos los que no habían dado la información correcta (Joseph, Interwiev Educateur Tana Novb, 2016).

- La salida del programa corresponde con el final del año y medio preestablecido. Con respecto a la atención a la diversidad afectivo-sexual, el recurso admite chicos homosexuales o transexuales, sin embargo se considera que se trata de una enfermedad que tiene que ser curada. Se exige a los chicos que, a lo largo de la intervención, se decidan entre ser hombre o mujer. Con respecto al tratamiento de otras manifestaciones de violencia, se reseñan maltratos en el seno familiar que se abordan con técnicas de mediación, así como un caso de violencia de género en la pareja de una de las jóvenes, aconsejando a la joven que intentase buscer una estabilidad con su pareja, proponiéndole que se casara para apaciguar la situación.

\subsection{Con respecto a las jóvenes}

Se han realizado tres entrevistas semiestructuradas con 3 antiguas beneficiarias del proyecto. Esto ha permitido abordar, en primer lugar, la historia de explotación de cada una de ellas y su experiencia en el recurso. Las tres refieren haber entrado por decisión propia y por motivos concernientes a la cobertura de las necesidades básicas. Las tres han ejercido la prostitución en la calle y en karaokes con clientes locales. El tiempo medio de ejercicio de esta actividad varía de 1 a 3 años. Con respecto a los motivos expresados para decidir abandonarla, las jóvenes argumentan diferentes razones: un embarazo adolescente, conocimiento de las acciones de la estructura de protección estudiada, a través del grupo de pares, y el estigma sufrido a causa del ejercicio de la prostitución junto con el deseo de ser reaceptada por la comunidad.

Con respecto a la experiencia en el programa, las tres se muestran ambivalentes; si bien 
afirman que su vida ha mejorado gracias al acceso a alternativas de trabajo diferentes a la prostitución, expresan también la falta de autonomía en la toma de decisiones vivida en el centro:

Yo quería estudiar, volver al colegio, siempre me ha gustado mucho estudiar, sabe...pero claro yo tenía mi bebe en camino...y bueno los educadores me dijeron que no era posible que volviera a estudiar, que ahora tenía que ganarme la vida para hacerme cargo de mi bebé...me dijeron que lo mejor era que me pusiera a montar un pequeño negocio de venta ambulante en la calle... yo no quería pero mi familia terminó por convencerme....ahora que el niño es más mayor me gustaría buscar dinero para intentar volver a la escuela (Kia, 2016).

Me enfadaba porque no podía gastar el dinero que me daban los educadores como necesitaba. Si tenía que comprarme una camisa para una entrevista de trabajo no me dejaban comprarla a mí, iban ellos y compraban la que ellos querían... Nos daban todo y luego nos quitaban de repente todo el dinero cuando el proyecto terminaba pero yo quería aprender a ahorrar y poder comprarme mis cosas como yo quiero (Gisela, 2016).

Cuando mi hijo nació fue un momento muy duro para mí en el proyecto... Tenía muchas dificultades para dejar al niño con alguien y en la asociación no me dejaban llevarlo a las entrevistas y tampoco cambiar los horarios según lo que necesitaba...tenía que ir cuando ellos decían, siempre." (Kia, 2016); "Cuando entré no tuve elección en lo que deseaba hacer, había dos opciones en el proyecto o hacer formación profesional o montar un negocio... decidí montar un negocio para tener cuanto antes dinero para poder hacer otra cosa... al final sigo donde empecé hace 4 años cuando acabé el programa, pero estoy contenta (Clarissa, 2016).

\section{Conclusiones}

\subsection{Con respecto al marco de trabajo}

El programa se limita a un análisis de la problemática basado en factores personales y familiares. Además, se analiza como un problema universal y atemporal, sin tener en cuenta el contexto y el momento histórico y social en el que tiene lugar. Esto impide una interpretación situada y la puesta en marcha de acciones adaptadas y va al encuentro de los modelos que analizan la explotación como una construcción cultural que puede ser cuestionada y modificada. (ONU, 1995)

Los factores utilizados para el análisis no se corresponden con ninguna de las conclusiones que manifiestan los estudios sobre las causas (ONU, 1995; Skrobanek, Boonpakdi y Janthakeero, 1997; Cole, 2010; Lafontaine, Tamez,M. y Caba, 2011; Orjuela López y Rodriguez Bartolomé, 2012), como tampoco coinciden con los causas expresadas por las jóvenes (C. 2016). Algunos de los factores expresados constituyen además una violación de los derechos fundamentales de los/las jóvenes en lo que se refiere, por ejemplo, a la no discriminación por razones de orientación o identidad sexual. Otros contribuyen a alimentar modelos familiares tradicionales -divorcio de los padres, familia monoparental-, modelos sexuales tradicionales -hombres con deseos irrefrenables y mujeres con conductas provocadoras-, a dejar en la sombra las diferentes relaciones de poder -clase social, raza, género-implicadas en la aparición y mantenimiento de la problemática y las diferentes violencias que pueden acompañar a la explotación en sí.

El problema se presenta de manera bien diferenciada a la prostitución de las adultas, a pesar de las diferentes conexiones existentes entre ambas realidades: entrada en la prostitución en la adolescencia y mantenimiento en la edad adulta (Szczepanick et al., 2014); consumo de prostitución como ejercicio de poder donde se consume a los y las más vulnerables, de las mujeres migrantes sin papeles a los y las adolescentes y niñas y niños (Gimeno, 2012).

La falta de un marco de trabajo claro y de una formación previa solida sobre la temática, tal y como aconsejan todos los documentos de trabajo con víctimas de violencia (OMS, 2001; Adams et al., 2009), fomenta también el mantenimiento de ciertos mitos y estereotipos entre el equipo, como el hecho de que las chicas no son capaces de salir de la situación o de que se trata de un trabajo fácil que les permite ganar mucho dinero en poco tiempo.

Todas estas interpretaciones dificultan la puesta en evidencia de los factores y causas profundas y macroestructurales que alimentan 
la problemática, lo que limita las acciones a llevar a cabo así como su eficacia tal y como han documentado otros estudios (Szczepanick et al., 2014). Además alimentan la puesta en marcha de acciones que no corresponden forzosamente con las necesidades y deseos de las jóvenes.

\subsection{Con respecto al programa de interven- ción}

Las dificultades, enunciadas con respecto al marco de interpretación y análisis en el punto anterior, se reflejan y tienen un impacto en la puesta en marcha de las acciones de intervención con los y las jóvenes.

La creación de un modelo de intervención que no tiene en cuenta la realidad, ni los conocimientos y las experiencias de las y los jóvenes a los que va dirigido ni tampoco el contexto donde se desarrolla, aumenta las dificultadas de salida de las personas implicadas, fomentando una autonomía parcial que no elimina completamente la vulnerabilidad y la precariedad material de la vida de los y las interesadas, lo que puede contribuir a que las chicas decidad volver a prostituise y a la entrada en la industria del sexo en la edad adulta (Ídem).

El análisis del problema como únicamente personal o/y familiar dificulta la puesta en marcha de la implicación de toda la comunidad en la construcción de una sociedad libre de explotación y refuerza el sentimiento de impunidad de los clientes que no son cuestionados en ningún momento. Deposita toda la responsabilidad de los hechos y de la recuperación en las y los jóvenes y en su entorno, pudiendo generar sentimientos de culpabilidad e impotencia que dificultan la movilización de las fortalezas y los recursos del y de la joven y de su entorno; y por lo tanto su salida de la situación de explotación.

Este abordaje impide que las jóvenes incorporen nuevos conocimientos con los que construir un sentido a su situación para así poder transformarla. El poder de interpretación de la situación y de la posible salida recae únicamente en los expertos y las expertas; yendo en contra de las recomendaciones del trabajo con víctimas de violencia establecidas por los marcos internacionales y por las organizaciones que intervienen con supervivientes (ONU, 1995; Skrobanek et al., 1997; OMS, 2001; Szczepanick et al., 2014)).
En última instancia estas interpretaciones se concretizan en una revictimización de las y los jóvenes. Prácticas, como las pruebas de virginidad o líneas de intervención donde la homosexualidad o la transexualidad se consideran enfermedades, refuerzan la imposibilidad de que los y las jóvenes puedan tener un control efectivo sobre su propio cuerpo y su propia sexualidad, con información adecuada para poder tomar la decisión que mejor les convenga, violando sus derechos. Estas prácticas vuelven a reforzar la idea de que son los y las expertas quienes detentan las claves de interpretación de la problemática, invalidando cualquier conocimiento derivado del joven o la joven, incluso sobre sí mismo/a (Ehrenreich y English, 2010)

La banalización y justificación de otras violencias sufridas por los y las jóvenes impide la reflexión sobre una comunidad libre de todo tipo de violencias, validándolas como formas aceptadas de relación y reforzando la indefensión aprendida en los y las jóvenes (ONU, 1995; OMS, 2001; Orjuela López y Rodriguez Bartolomé, 2012).

Finalmente, la propuesta de soluciones basadas únicamente en la puesta en marcha de alternativas económicas impide que los y las jóvenes tengan una asistencia jurídica, en caso de que deseen denunciar la situación, reforzando el círculo de impunidad y legitimando la idea de que la explotación es solo un tipo de trabajo más, así como poder decidir qué alternativas desean para su vida, debilitando su capacidad de decisión y de autonomía; en contra igualmente de las recomendaciones existentes en materia de trabajo con víctimas de violencia (ONU, 1995; OMS, 2001).

\section{Recomendaciones}

Siguiendo el modelo de análisis propuesto al principio, y tras la revisión de los resultados obtenidos durante la investigación, es necesaria una reflexión sobre cómo poner a los y las jóvenes en el centro y ofréceles alternativas viables y sostenibles de autonomía. Las prácticas analizadas permiten pequeños cambios, pero no interpelan ni al contexto ni a las causas profundas; sitúan a los y las jóvenes en situaciones de indefensión y dependencia del recurso y reproducen unos modelos de relación no igualitarios ni democráticos. En este sentido el Trabajo Social debería ser capaz de comprender 
el rol que cumple en un momento determinado la explotación, en una sociedad determinada, para adaptar las políticas y las estrategias de intervención y, mientras se trabaja para combatir las causas macroestructurales que legitiman y permiten el surgimiento de esta problemática, poder dar una respuesta adaptada a las chicas y chicos que se encuentran en situación de explotación y que desean realizar un cambio en este sentido en sus vidas.

Así, las siguientes recomendaciones vendrían a aportar ciertas pistas en este sentido:

Revisar el marco de interpretación de trabajo y nutrirlo con conocimientos y datos basados en evidencias científicas, que respeten los derechos fundamentales de los y las jóvenes; realizar una revisión de la literatura existente sobre el tema, así como los marcos de trabajo con víctimas de violencia; y realizar un análisis de contexto sobre la naturaleza de la problemática que incluya los conocimientos y las voces de los y las jóvenes implicadas.

Las acciones que se habrían de poner en marcha pasarían por implementar actividades de formación permanente de los equipos, incluyendo una supervisión de casos mensual, basada en los indicadores de seguimiento establecidos gracias a las dos acciones preliminares; crear un modelo de intervención integral que tenga en cuenta todos los aspectos implicados en el apoyo a las y los jóvenes, que tenga como principios la protección y la lucha contra todo tipo de violencia y la superación de desigualdades, y como objetivo la reducción de la vulnerabilidad y la puesta en marcha de alternativas de vida sostenibles en el tiempo para los y las jóvenes, acorde a sus deseos y necesidades; crear herramientas de trabajo adaptadas a los resultados obtenidos en los dos puntos anteriores, que se ajusten a los estándares mínimos de trabajo con víctimas y de respeto de los derechos humanos; y poner en marcha espacios de reflexión grupal donde tener acceso a información sobre las causas estructurales que se encuentran detrás de la explotación y que permitan a los y las jóvenes tejer un sentido sobre su experiencia.

El objetivo final de estas pistas de trabajo sería poder garantizar unos espacios de seguridad y de no estigmatización para los y las jóvenes, donde se tejan posibilidades y se trasformen desigualdades.

\section{Referencias bibliográficas}

Adams, A., Ahrens, C., Campbell, R., Self, T. y Wasco, S. (2009). Training Interviewers for Research on Sexual Violence A Qualitative Study of Rape Survivors' Recommendations for Interview Practice. Violence against women, 15(5), 595-617.

ATTAC. (2009). Mondilaisation de la prostitution, atteinte contre la dignité humaine. Clamecy: La Nouvelle Imprimerie Laballery.

Bautista, O. (2016). Interwiev Educateur-Novb 2016 [Recorded by Joseph]. Antananarivo, Madagascar.

Bidaseca, K. y Vazquez Laba, V. (2011). Feminismos y decolonilaidad. descolonizando el feminismo desde y en América Latina. Buenos Aires: Ediciones Godot.

Buisset, A. y Doppler, B. (2013). Le tourisme sexuel implicant les enfants à Madagascar. Ampleur du phénoméne et analyses des mécanismes de signalement. Antananarivo: Ecpat France.

Bustamante, A. (2010). Mapa de utopías. Propuesta pedagógica para la prevención de la explotación sexual comercial desde la adolescencia. Madrid: Instituto Universitario IEPALA-UCM "Rafael Burgaleta".

C.G.K. (2016). Histoires de vies des survivantes. (O. B. Cosa, Interviewer), 23 de Noviembre.

Clarissa. (2016). Interwiv jeune survivante 2. (O. Bautista Cosa, Interviewer). Noviembre.

Cole, J. (2010). Sex and Salvation. Imagining the future in Madagascar. Chicago: The University of Chicago Press.

ECPAT International, U. (1996). Declaración de la reunión realizada en Estocolmo con motivo del Congreso Mundial contra Explotación Sexual Comercial de los Niños. Estocolmo: ECPAT International. Retrieved from: http://www.ecpat-spain.org/imagenes/tablaContenidos01sub/Plan\%20de\%20Accion-Declaracion-Estocolmo.pdf

Ehrenreich, B. y English, D. (2010). Por tu propio bien. 150 años de consejos expertos a mujeres. Madrid: Capitan Swing.

ERIC. (2013). Ethical Reaserch Involved Children. Recuperado de: childethics.com: http://childethics.com/ 
Europa, A.P. (2011). Manual para Parlamentarios : Convenio del Consejo de Europa para la Protección de los niños contra la Explotación Sexual y el abuso sexual. (Convenio de Lanzarote). Estraburgo: Secretariado de la Comisión de Asuntos Sociales, de Salud y Familiares. Recuperado de: http://www.fapmi. es/imagenes/subsecciones1/1de5_Doc_03_Convenio\%20Lanzarote_Parlamentarios.pdf

Gimeno, B. (2012). La Prostitucion. Apuntes para un debate abierto. Barcelona: Edicions Bellaterra.

Gisela. (2016). Interwiev Jeune survivante 1. (O. Bautista Cosa, Interviewer). Noviembre.

Gómez, J., Latorre, A., Sánchez, M. y Flecha, R. (2006). Metodología Comunicativa Critica. Barcelona: El Roure.

Inoccenti, C. d. (2012). La seguridad de los niños en línea.REtos y estrategias mundiales . Florencia: UNICEF.

ISA. (2001). isa-sociology.org. Recuperado de: www.isa-sociology.org: http://www.isa-sociology.org/es/ sobre-isa/codigo-etico-440/

Jabardo, M. (. (2012). Feminismos Negros. Una antología. Madrid: Traficantes de Sueños.

Joseph. (2016). Interwiev Educateur Tana. (O. Bautista Cosa, Interviewer) Noviembre

Kia. (2016). Interwiev Jeune Survivante 3. (O. Bautista Cosa, Interviewer). Noviembre.

Lafontaine, E., Tamez,M. y Caba, A. (2011). Por la sostenibilidad ética, social y cultural. Manual para la Prevención de la Explotación Sexual Comercial de Niños, Niñas y Adolescentes desde el Sector Turismo. Santo Domingo: UNICEF-Republica Dominicana.

Lagarde y de los Ríos, M. (1990). Los cautiverios de las mujeres. Madresposas, monjas, putas, presas y locas. Madrid: Editorial Horas y horas.

Levin, L. y Peled, E. (2011). The Attitudes Toward Prostitutes and Prostitution Scale: A New Tool for Measuring Public Attitudes Toward Prostitutes and Prostitution. Research on social work practice, 582-593.

Maina Wanjiru, C. (2016). The African Committee of Experts on the Rights and Welfare of the child (ACWRC)-SDGS and Agenda 2063. Dar Es Salaam-Tanazania.

Observatorio de la Infancia. (2010). III Plan de Acción contra la Explotación Sexual de la Infancia y la Adolescencia- 2010/2013. Madrid: Ministerio de Sanidad, Politica Social e Igualdad. Recuperado de: http://www.observatoriodelainfancia.msssi.gob.es/productos/pdf/IIIPESIDefinitivo.pdf

OHCR. (2011). Observación General $N^{o}$ 13: Derecho del niño a no ser objeto de ningun tipo de violencia. Ginebra: Naciones Unidas-Comité Derechos del Niño. Retrieved from: http://www2.ohchr.org/english/ bodies/crc/docs/CRC.C.GC.13_sp.pdf

OMS. (2001). Putting Women First. Ethical and safety recomnendations for resaerch on domestic violence against women. Ginebra: Department of Gender and Women's Health. Retrieved from: http://www. who.int/gender/violence/womenfirtseng.pdf

ONU. (1995). Declaración de Beijing. Cuarto Conferencia Mundial sobre la Mujer (pp. 89-90). Pekin: Naciones Unidas. Recuperado de: http://www.unwomen.org/ /media/headquarters/attachments/sections/ csw/bpa_s_final_web.pdf

Orjuela López, L. y Rodriguez Bartolomé, V. (2012). Violencia sexual contra los niños y niñas. Abuso y explotacion sexual infantil. Guía de material básico para la formación de profesionales. Madrid: Save The Children España.

Ravaozanany, N., Rakotoarindrasata, M., De Dieu, J., Rabenoro, M. y Rasendrasoa, L. (2012). De la SADC et le dévèloppement 2012. Barometre Madagascar. Johannesburg: Gender Links.

Ricaldi Coquellin, A.M. (2010). Visages d'exclusion dans la société malgache contemporaine. París: L'Harmattan.

Sassen, S. (2003). Contrageografías de la Globalización. Género y cuidadania en los circuitos transfronterizos. Madrid: Traficantes de sueños.

Skrobanek, S., Boonpakdi, N. y Janthakeero, C. (1997). Tráfico de mujeres. Realidades Humanas en el negocio internacional del sexo. Madrid: Ediciones Narcea S.A.

Szczepanick, G., Ismé, C. y Boulebsol, C. (2014). Rapport de recherche: Connaître les bésoins des femmes dans l'industrie de sexe pour mieux baliser les services. Québec: Concertation des luttes contre l'exploitation sexuelle (CLES).

UNDP. (2015). Human Developpement Data (1980-2015). Recuperado de: Human Developpement Data (1980-2015): http://hdr.undp.org/en/composite/HDI 